\title{
Effects of Birefringence on the Electromagnetic Guidance of Structures Produced by Femtosecond Laser
}

\author{
Fernanda Mantuan Dala Rosa de Oliveira ${ }^{1}$, Ismael Chiamenti ${ }^{1}$, José Luís Fabris ${ }^{1}$, Marcia Muller ${ }^{1}$, \\ Hypolito José Kalinowski ${ }^{2}$ \\ ${ }^{1}$ Universidade Tecnológica Federal do Paraná, Curitiba, PR, Brazil e-mails: fmantuan@gmail.com, \\ Ismael.utfpr@gmail.com,fabris@utfpr.edu.br,mmuller@utfpr.edu.br \\ ${ }^{2}$ Universidade Federal Fluminense, Niterói, Brazil, e-mail: hjkalinowski@id.uff.br
}

\begin{abstract}
Analysis of the state of polarization of light guided by structures recorded in lithium niobate and lithium fluoride crystals indicates that the recording method is determinant in the formation of birefringent structures. Each structure was written with a single and continuous translation of the crystal transversally to the laser beam. Birefringence was observed in the structures recorded in both crystals, despite the lack of intrinsic birefringence in lithium fluoride. The recorded structures are anisotropic showing regions with different birefringence. The structure generated with femtosecond laser is directly linked to the recording method and the crystalline lattice. This characteristic can be advantageous for application in photonic devices.
\end{abstract}

Index Terms - Lithium Fluoride, Lithium Niobate, State of Polarization, Waveguide Femtosecond Writing.

\section{INTRODUCTION}

The inscription of structures in dielectric materials by exposure to ultrafast laser pulses was described in 1996 [1]. An advantage of this method is the modification of the refractive index only in the region of the material exposed to the focused energy of the laser, making possible the creation of three-dimensional structures [2]. Different types of dielectrics, such as glasses, crystals or polymers, can be used to manufacture photonic devices as it is a nonchemical method [3]-[4]. However, the bulk material must have adequate characteristics for the intended applications, such as broad band range of transparency or high electro-optic coefficients [5]. Tightly focused femtosecond laser pulses can cause multiphoton and electron impact ionization into the material. These two processes are the main ones responsible by the material breakdown and consequent formation of a microplasma. According to ref [6], two types of damage were produced in $\mathrm{LiNbO}_{3}$ by the incidence of fs laser. Under low laser fluences, localized defects created in the crystalline lattice cause an increase in the extraordinary refractive index and can be annealed at moderate temperatures. On the other hand, high laser fluences focused in the crystal produce localized lattice distortions leading to a volume increase and a refractive index decrease. The material surrounding region is affected by the structural modification and is subjected to a stress-induced birefringence. In this scenario, beam defocusing by the microplasma competes with the self-focusing leading to laser pulses filamentation. In this sense, the 
unique properties of the lithium niobate crystal $\left(\mathrm{LiNbO}_{3}\right)$ have been exploited. It is a crystal with high Curie temperature, excellent optical transparency in the visible and infrared wavelengths, high electro-optic coefficients and nonlinear optical susceptibility [7]. This material has a trigonal crystal structure formed by planes of oxygen atoms in a distorted hexagonal configuration. The octahedral interstitials formed in the structure are one-third filled by lithium ions, one-third by niobium ions and one-third are empty. $\mathrm{LiNbO}_{3}$ is a naturally birefringent crystal with characteristics that justifies its wide use in acoustic wave transducers, acoustic delay lines, acoustic filters, amplitude and optical phase modulators, second harmonic generators, Q-switches, beam deflectors, phase conjugators, dielectric waveguides, memory elements, holographic data processing devices, among other devices [8]. Particularly, erbium doped $\mathrm{LiNbO}_{3}$ crystals allowed the development of an entire class of new waveguide devices such as modulators, efficient wavelength filters and adjustable lasers [9]. Additionally, it is an excellent material for integrated optics [10]. However, the structure of the $\mathrm{LiNbO}_{3}$ has intrinsic defects, which make the material very prone to optical damage when irradiated by high powers of visible light. In this case, $\mathrm{MgO}$ has been successfully used as doping agent to decrease the photorefractive effect in this class of crystals [11].

Femtosecond laser pulses have been recently used to produce waveguides in $\mathrm{LiNbO}_{3}$ crystals [12][18]. Structures were produced for high confinement at $1550 \mathrm{~nm}$ in z-cut [14], as well as in x-cut crystals [15], but disappeared after approximately 30 and 100 days, respectively. The characteristics of the waveguides depend on the parameters of the writing system. The so-called type I waveguides associated to the refractive index increase were generated in $\mathrm{z}$ - and $\mathrm{x}$-cut $\mathrm{LiNbO}_{3}$ crystals by the incidence of fs laser with pulse energy below $0.5 \mu \mathrm{J}$, as well as in $\mathrm{Er}^{3+}, \mathrm{MgO}$ codoped $\mathrm{LiNbO} 3$ with pulse energies from $0.54 \mu \mathrm{J}$ to $0.66 \mu \mathrm{J}$. In the later case, guiding in the infrared region occurred only for the TM mode propagation [16]. Type I waveguides were reported to vanish when annealed at $150{ }^{\circ} \mathrm{C}$ for $1.5 \mathrm{~h}$. On the other hand, pulse energy above the damage threshold produces Type II waveguides [17] that were not subjected to thermal decay at $150{ }^{\circ} \mathrm{C}$ for several hours. Guidance in type II structures occurs along the stress region formed around the laser focus region of decreased refractive index. Depending on the crystal orientation, both structures can be formed at different positions along the laser beam path.

Another crystal of interest for the production of waveguides is the lithium fluoride ( $\mathrm{LiF}$ ), an alkaline halide material of unique properties. Unlike the other halides, LiF crystal presents very low hygroscopy, has high coefficient of hardness, and its cleavage is difficult. The LiF crystal has cubic face centered crystal structure, is optically inactive and exhibits high optical transparency for wavelengths ranging from $\sim 150 \mathrm{~nm}$ to above $6,000 \mathrm{~nm}$ [19]. Color centers can be produced in the material by irradiation with electrons, $\mathrm{X}$-rays or $\gamma$-rays, although additive coloring is not feasible. The F-center has absorption band in the ultraviolet region, with peak at $248 \mathrm{~nm}$ without an identified emission band. The main luminescence of color centers in $\mathrm{LiF}$ is associated with aggregated $\mathrm{F} 2$ and F3+ centers, with bands located at $678 \mathrm{~nm}$ and $535 \mathrm{~nm}$ [20], respectively, under excitation of the Brazilian Microwave and Optoelectronics Society-SBMO received 15 Dec 2017; for review 19 Dec 2017; accepted 26 Apr 2018 Brazilian Society of Electromagnetism-SBMag 
superimposed absorption bands, called the $\mathrm{M}$ band $(\sim 450 \mathrm{~nm})$. Fluorescence of these aggregated centers was also observed by optically pumping the F band. Studies on the equilibrium dynamics of the aggregate centers showed the possibility of provide a selective depopulation of one of the species [21]. The efficiency of the lithium fluoride optical pumping cycle allows a number of applications such as waveguides [22]-[27], color center lasers [28], including those with distributed feedback [29] periodic photorefractive structures [30] and even radiation detectors [31]. Radiation detectors are particularly interesting for imaging with confocal laser-scanning-microscopy using fluorescent excitation, allowing better resolution than conventional techniques and greater applicability in samples.

In the development of color centers lasers in LiF crystals, external cavities formed by mirrors and also distributed feedback structures based on color centers in an original crystal (pristine) have been used. Laser emission from approximately $530 \mathrm{~nm}$ to $1300 \mathrm{~nm}$ [28], [32]-[33] has been obtained.

Adequate modifications in the parameters of the writing technique allowed the fabrication of Bragg waveguides in LiF crystals with femtosecond laser pulses. Several works focused on the study of the Bragg waveguide dimensions and the associated refractive index variations [25], the measurement of light propagation parameters in the range from $458 \mathrm{~nm}$ to $1550 \mathrm{~nm}$ [26]-[27] and the production of LiF crystal waveguide lasers [24], [33].

In $\mathrm{LiF}$ and $\mathrm{MgO}$ single crystals, lattice distortions produced by femtosecond laser were investigated by the observation of distributions of birefringence around the photoexcited region [34]. Birefringence was associated to the structural changes caused by strain and stress. The photoexcitation with a femtosecond laser produces a stress wave associated with the nonlinear ionization at the focal region. Consequently, cracks and dislocations are expected to appear in the crystal structure as a result of the thermal stress around the photoexcited region.

Despite the efforts, there are still some features concerning to the optical guidance of structures produced in $\mathrm{LiF}$ and $\mathrm{LiNbO}_{3}$ that must be investigated in order to produce optimized optical devices and lasers. Laser emission in $\mathrm{LiF}$, for example, can be improved with an investigation of the polarization of the light guided by the waveguides. On the other hand, the crystal cut and the writing parameters influence the guiding properties of structures produced in $\mathrm{LiNbO}_{3}$.

In this work, were studied guidance properties of structures recorded in $\mathrm{LiNbO}_{3}$ and $\mathrm{LiF}$ crystals according to the state of polarization of the coupled light.

\section{EXPERIMENTAL}

Guiding structures were produced in $\mathrm{LiF}$ and $\mathrm{LiNbO}_{3}: 0.3 \% \mathrm{Er}_{2} \mathrm{O}_{3}: 0.5 \% \mathrm{MgO}$ crystals by a regenerative amplifier based on Ti:Sapphire (Coherent, Libra-F, at $800 \mathrm{~nm}$ with pulses of $140 \mathrm{fs}$ at a repetition rate of $1 \mathrm{kHz}$ ) focused by a 20x microscope objective lens (Thorlabs, LMH-20x, $1064 \mathrm{~nm}$, $0.4 \mathrm{NA}$ ). $\mathrm{LiNbO}_{3}$ crystals were cut and polished to eliminate scattering effects on the surface. A circular filter of linear neutral density was used to set the intensity of the laser beam during the 
recording process. Each structure was written with a single and continuous translation of the crystal transversally to the laser beam. Depending on the relative speed, a continuous volume of modified refractive index that characterizes the waveguide is formed inside the crystal. The recording parameters for the LiF crystal were laser energy of $5 \mu \mathrm{J}$ per pulse, focalization depth of $100 \mu \mathrm{m}$ from the top surface and translation speed of $191 \mu \mathrm{m} / \mathrm{s}$. For the $\mathrm{LiNbO}_{3}$ crystal the translation speed was 50 $\mu \mathrm{m} / \mathrm{s}$ with a laser energy of $2 \mu \mathrm{J}$ focalized at $100 \mu \mathrm{m}$ from the surface.
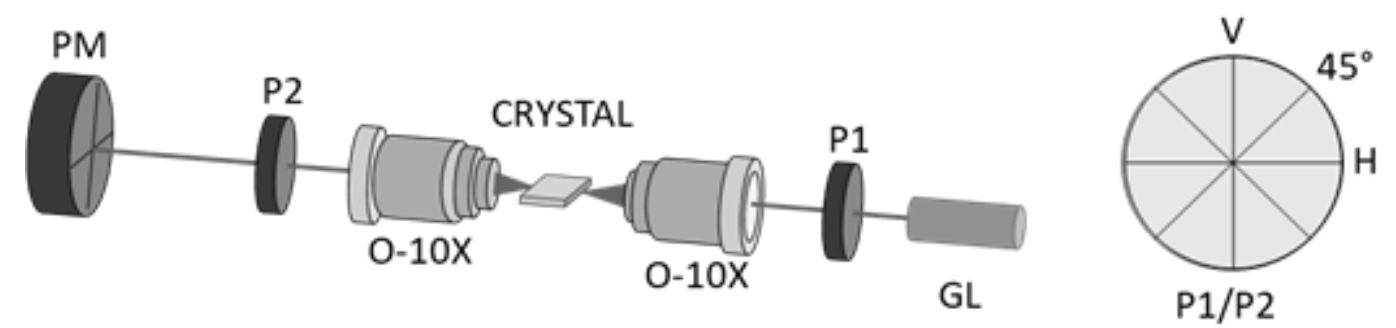

Fig. 1. Schematic diagrams: (left) Experimental set-up for detecting the beam profile at different polarizations; (right) Orientations for the transmission axis of the $\mathrm{P} 1$ and $\mathrm{P} 2$ polarizers.

Fig. 1 shows the experimental setup used to measure the beam profile of the light emerging from the structures. The light of a semiconductor laser at $635 \mathrm{~nm}$ collimated by a GRIN lens (GL) is focused at the crystal surface by a microscope objective (O-10X) and coupled to the waveguide. A second microscope objective $(\mathrm{O}-10 \mathrm{X})$, positioned at the focal distance from the opposite face of the crystal, collects the light emerging from the waveguide. The laser beam is then captured by a Profile Meter (PM) (Thorlabs, BP104-UV, wavelength range from 200 to $1100 \mathrm{~nm}$ ) positioned in the collimated region of the laser beam. A XYZ (Newport, ULTRAlign 561D-XYZ) stage provides the optical alignment of the lenses and a XY stage (Newport, ULTRAlign 561D-YZ) is used for positioning the crystal. The data acquisition system detects light intensities along the vertical and horizontal axes. Images of the beam profile are then reconstructed by software (Thorlabs Beam 3.0 for $\mathrm{LiF}$ and 6.2 for $\mathrm{LiNbO}_{3}$ images) from the intensity profiles collected by the Profile Meter. The state of polarization (SOP) of the light emerging from the waveguide was analyzed with the help of two polarizers ( $\mathrm{P} 1$ and $\mathrm{P} 2$, extinction ratio of 1:4,000). The transmission axis of $\mathrm{P} 1$ assumed only two orientations, vertical $(\mathrm{V})$ or horizontal $(\mathrm{H})$. For each orientation of $\mathrm{P} 1$, the beam profile was recorded in the absence of $\mathrm{P} 2(\phi)$ and also for the $\mathrm{P} 2$ transmission axis parallel $(\mathrm{VV})$, perpendicular $(\mathrm{VH})$ and at $45^{\circ}\left(\mathrm{V} 45^{\circ}\right)$ with the $\mathrm{P} 1$ axis.

\section{RESULTS}

The cross-sectional transmission microscopy image of the structure recorded in $\mathrm{LiNbO}_{3}$ shows dark regions in the center of the figure indicated by arrows in Fig. 2 (a). Additionally, two lines in the longitudinal image of Fig. 2 (b), top view, indicate the recording of two parallel structures.

These dark regions are related to the decrease in the refractive index of the material resulting from self-focusing with laser power above the threshold for collapse. The bright regions localized around Brazilian Microwave and Optoelectronics Society-SBMO received 15 Dec 2017; for review 19 Dec 2017; accepted 26 Apr 2018 Brazilian Society of Electromagnetism-SBMag 
the dark ones, result from stress-induced changes that increases the refractive index (Type II waveguide) [17].
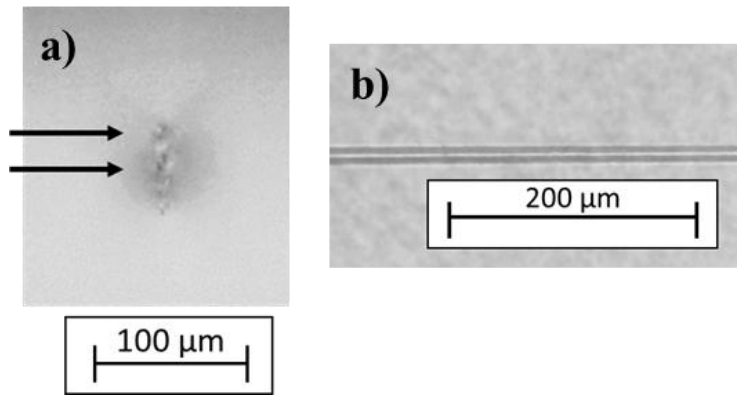

Fig. 2. Images of the structure recorded in $\mathrm{LiNbO}_{3}$ captured by transmission microscopy: (a) cross-sectional image of the crystal showing the structure of approximately $50 \mu \mathrm{m}$ of width; (b) image of the structure formed by double lines in a top view of the crystal.

As $\mathrm{LiNbO}_{3}$ crystal is birefringent, changes in refractive index can occur in both the ordinary and the extraordinary indexes. Structural changes, created in a regime of moderate recording intensity, increase the extraordinary refractive index whereas the ordinary refractive index decreases. On the other hand, nonlinear absorption effects at high energy pulses causes heavy damage to the material, reducing both refractive indexes and resulting in the dark area. Besides, a stress-induced birefringent region is formed around the damaged region [5],[6]. A critical power for breakdown in $\mathrm{LiNbO}_{3}$ of 0.3 MW corresponding to an energy of $0.12 \mu \mathrm{J}$ was reported [6]. Therefore, as the energy per pulse of 2 $\mu \mathrm{J}$ used to produce the structures in $\mathrm{LiNbO} 3$ is higher than the critical energy for nonlinear ionization, filamentation caused by a dynamic balance between self-focusing and plasma defocusing is expected to occur.

Fig. 3 shows images of the structure recorded in LiF captured by transmission microscopy. It can be seen in Fig. 3 (a) the elongated transverse profile of the structure, which results from the expected filamentation due to the recording method [5]. Unlike $\mathrm{LiNbO}_{3}$, the dark region in the longitudinal profile of the LiF, Fig. 3 (b), exhibits a single line corresponding to the inscribed waveguide.
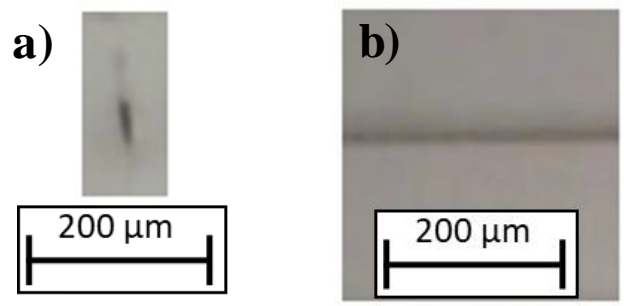

Fig. 3. Images of the structure inscribed in LiF captured by transmission microscopy (a) cross-sectional image, produced with a laser beam penetrating the crystal from the top surface (b) longitudinal image of the structure formed by a single line in a top view of the crystal.

In the recording method, the laser beam was launched on the upper surface of the crystal that also corresponds to the upper side of the cross-sectional images of Fig. 2 and 3. 
a) $\mathrm{V} \phi \mathrm{LiNbO}_{3}$

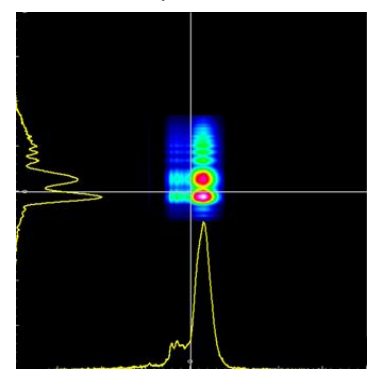

c) $\mathrm{V} \phi \mathrm{LiF}$

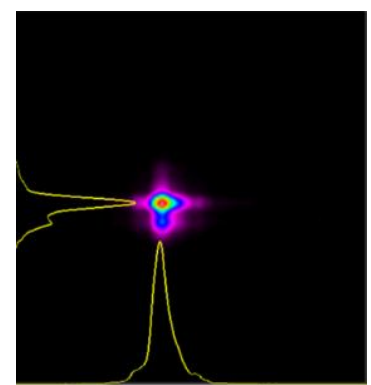

b) $\mathrm{H} \phi \mathrm{LiNbO}_{3}$

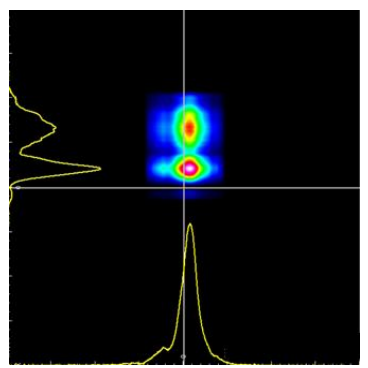

d) $\mathrm{H} \phi \mathrm{LiF}$

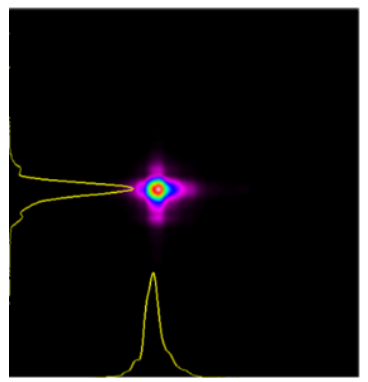

Fig. 4. Reconstructed images of the near field profile of the beam emerging from the structure inscribed in $\mathrm{LiNbO}_{3}(\mathrm{a}, \mathrm{b})$ and in $\mathrm{LiF}(\mathrm{c}, \mathrm{d})$, for the $\mathrm{SOP}$ of the incident light at $\mathrm{V}$ and $\mathrm{H}$ directions.

Reconstructed images that represents the near field profile of the beam emerging from the structures inscribed in $\mathrm{LiNbO}_{3}$ and $\mathrm{LiF}$ are shown in Fig. 4. These images correspond to the profiles obtained by controlling only the SOP of the incident light, and therefore, P2 was removed from the setup. Polarization of light transmitted by $\mathrm{P} 1$ was at the $\mathrm{V}$ or $\mathrm{H}$ directions. The orientations of the images in Fig. 4 (a), (b) and 4 (c), (d) are equivalent to the actual spatial orientation of the crystals in Fig. 2 (a) and Fig. 3 (a), respectively. In other words, the upper side of the images corresponds to the upper surface of the crystal. Fig. 4 (a) and 4 (b), exhibit in the vertical axis an elongated profile along the crystal depth composed of two main regions of guidance. The profile in the horizontal axis shows corresponds to the light guided by the whole structure. Nevertheless, modifications in the SOP of the incident light shifts the xy position of these regions.

The reconstructed images of Fig. 4 (c) and 4 (d), exhibits a near field profile for the LiF with a more regular shape comprised of one main region of maximum of intensity, therefore, a single waveguide. Beam profiles of the light guided by the structure in $\mathrm{LiNbO}_{3}$ obtained for $\mathrm{P} 1$ and $\mathrm{P} 2$ transmission axes at different orientations are shown in Fig. 5 and 6. For P1 at V or $\mathrm{H}$ directions, $\mathrm{P} 2$ was changed from the $\mathrm{V}, 45^{\circ}$ and $\mathrm{H}$ directions or was removed $(\phi)$. 

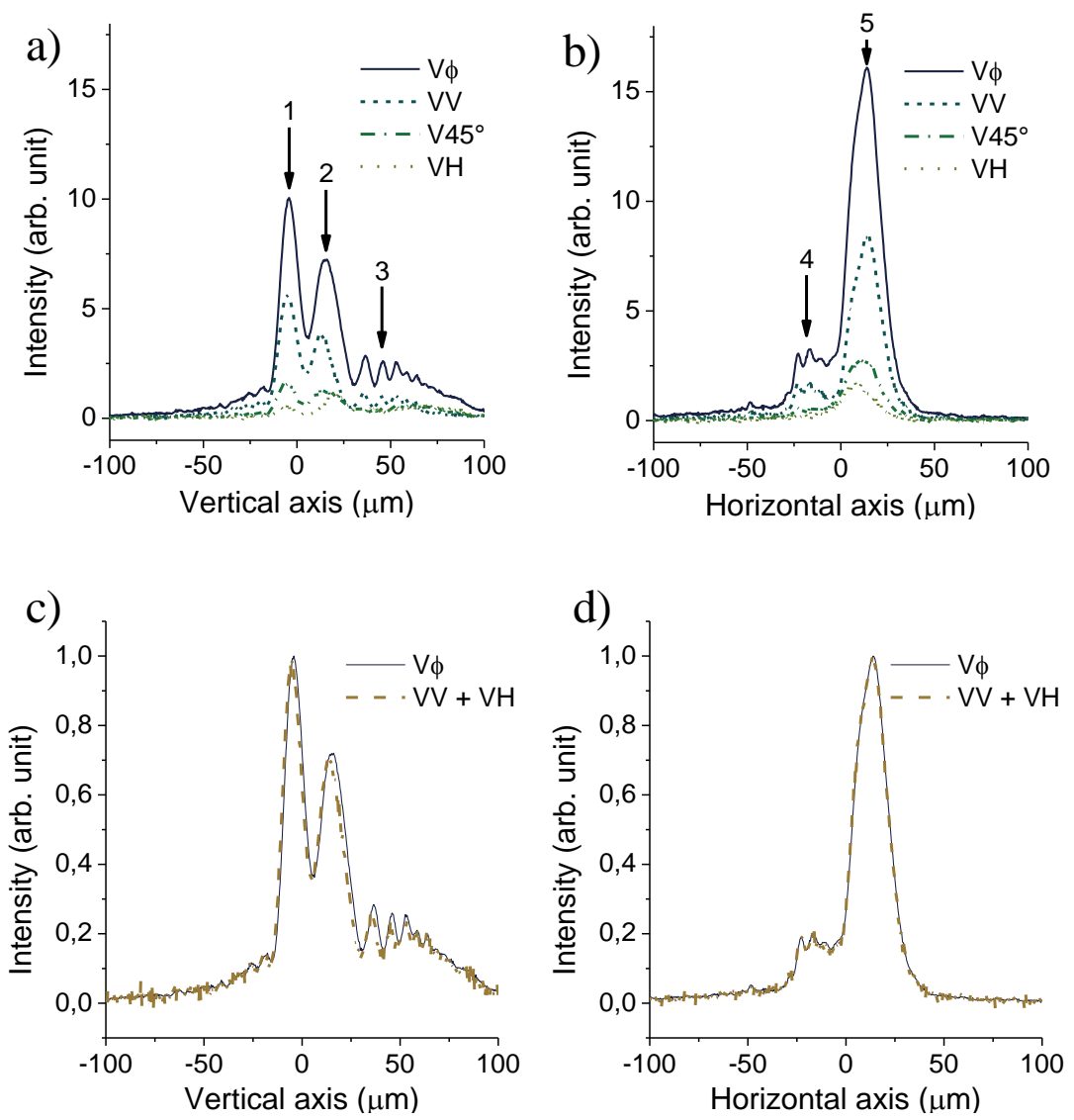

Fig. 5. (a, b) Graphical representations of the near field profile of the beam emerging from the structure inscribed in $\mathrm{LiNbO}_{3}$ for P1 at V direction and P2 absent or at V, 45 and H directions; (c, d) Signal obtained with P1 (V) and without P2 (Ф) compared with the combination of the signals obtained for $\mathrm{VV}$ and $\mathrm{VH}$.

For all P1 and P2 arrangements, the intensity and shape of the beam profile remained constant as can be seen in Fig. 5 (c) and 5 (d). In Fig. 5 (a), the profile on the vertical axis (crystal depth) for P1 aligned at vertical and P2 absent (V申), exhibits three peaks, also shown in Fig. 4 (a). Peak 1, corresponds to the guidance at the innermost region of the structure in the crystal which suffers the largest decrease of refractive index due to the laser focusing during the inscription. In the region near to the crystal surface occurs a leakage of the guided power to the external medium, peak 3 of Fig. 5 (a). When the transmission axes of both polarizers are aligned (VV), the overall intensity of the signal decreases but the profile still exhibits the 3 peaks. For the $\mathrm{V} 45^{\circ}$ arrangement, the signal decreases further, peak 3 almost vanishes and the profile shows only peaks 1 and 2 with approximately the same intensity. When P1 and P2 directions are orthogonal (VH) an output signal is still detected. Peak 1 becomes less intense than peak 2 . Therefore, the guidance shifts towards the surface of the crystal.

The beam profile in the horizontal axis, Fig. 5 (b), exhibits an intense peak 5, corresponding to the combined intensity of the three peaks (1,2 and 3) shown in the vertical axis. For $\mathrm{V} \phi$ and $\mathrm{VV}$ arrangements, is observed a lateral lobe 4 which vanishes for $\mathrm{V} 45^{\circ}$ and $\mathrm{VH}$. The horizontal and the vertical profiles experimentally obtained for $\mathrm{V} \phi$, Fig. 5 (c) and 5 (d), are reconstructed through the addition of the profiles obtained for $\mathrm{VV}$ and $\mathrm{VH}$ configurations. Each anisotropic region of the structure produced by the femtosecond laser has a particular birefringence. Therefore, for a linearly 
polarized incident light, each region of guidance propagates the field components in the directions of its birefringence axes. As a consequence, the shape of the beam profile changes for different orientations of the $\mathrm{P} 2$ transmission axis.
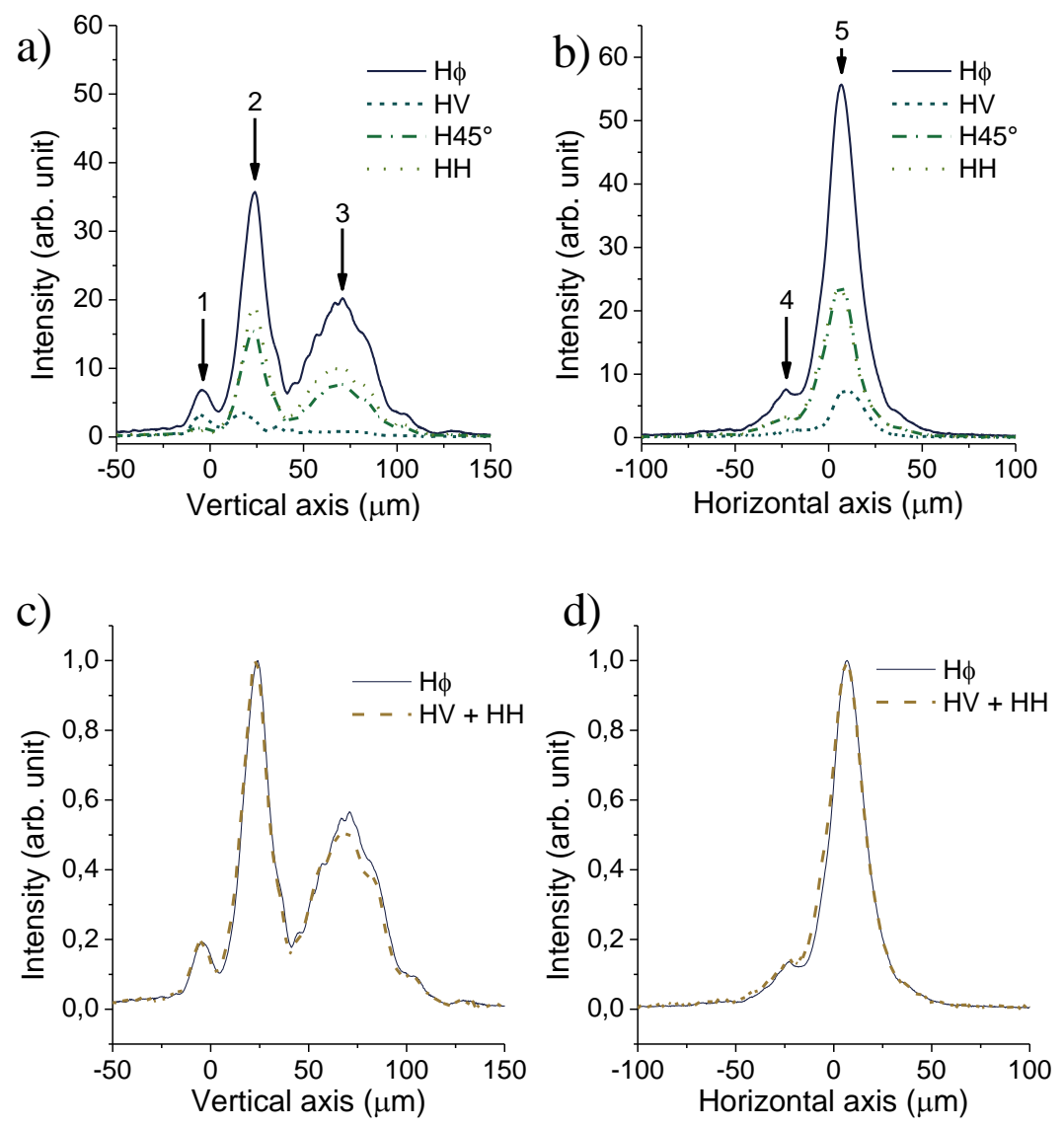

Fig. 6. (a, b) Graphical representations of the near field profile of the beam emerging from the structure inscribed in $\mathrm{LiNbO}_{3}$ for $\mathrm{P} 1$ at $\mathrm{H}$ direction and $\mathrm{P} 2$ absent or at $\mathrm{V}, 45^{\circ}$ and $\mathrm{H}$ directions; (c, d) Signal obtained with P1 (H) and without P2 (Ф) compared with the combination of the signals obtained for $\mathrm{HV}$ and $\mathrm{HH}$.

In Fig. 6 (a), the beam profile obtained for $\mathrm{H} \phi$ configuration also shows three peaks. However, unlike profile obtained for $\mathrm{V} \phi$, peaks 2 and 3 are the most intense. In this case, light preferentially propagates in the guidance regions nearly to the surface of the crystal. For the $\mathrm{H}_{4} 5^{\circ}$ and $\mathrm{HH}$ configurations, peak 1 vanishes. For HV, peaks 1 and 2 have approximately the same amplitude and peak 3 vanishes. Therefore, the innermost portion of the structure produced in the crystal guides preferentially light linearly polarized at the vertical direction. On the other hand, the region nearly to the surface guides preferentially light linearly polarized at the horizontal direction. Additionally, leakage of the light occurs preferentially for the horizontal polarization.

The beam profile at the horizontal axis, Fig. 6 (b), shows peak 5 which corresponds to the combined intensity of the three peaks in the vertical axis (Fig. 6 (a)). For this case, the lateral lobe 4 decreases for $\mathrm{HV}$ and $\mathrm{HH}$, and vanishes just for $\mathrm{V} 45^{\circ}$.

For incident light linearly polarized at the horizontal direction, the beam profile obtained without P2 is reconstructed by the addition of the profiles for $\mathrm{HV}$ and HH, as shown in Fig. 6 (c) and 6 (d). 

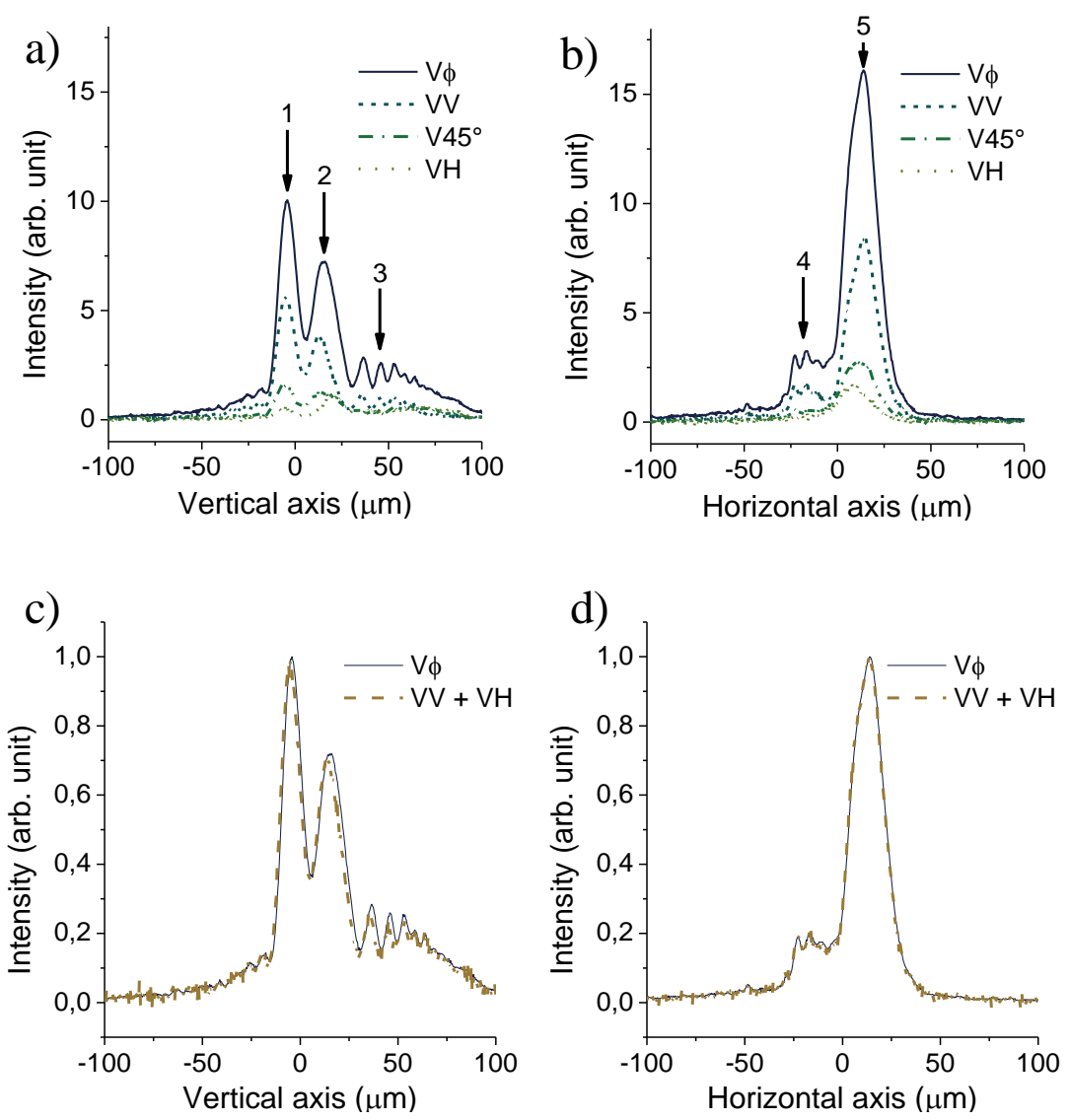

Fig. 7. (a, b) Graphical representations of the near field profile of the beam emerging from the structure inscribed in LiF

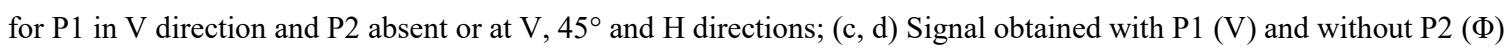
compared with the combination of the signals obtained for $\mathrm{VV}$ and $\mathrm{VH}$.

Beam profiles of Fig. 7 and 8 reveal the induced birefringence of the structure inscribed in lithium fluoride crystal. As it can be seen, guidance is obtained even for orthogonal directions of P1 and P2 transmission axes, VH arrangement.

Fig. 7 (a), shows the beam profile at the vertical axis composed of two main peaks. As peak 2 is the most intense, guidance occurs preferentially in the region of the inscribed structure close to the crystal surface for $\mathrm{V} \phi, \mathrm{VV}$ and $\mathrm{V} 45^{\circ}$ configurations. However, for $\mathrm{VH}$, guidance occurs in the innermost region of the crystal. In the horizontal axis, the beam profile for $\mathrm{V} \phi, \mathrm{VV}$ and $\mathrm{V} 45^{\circ}$ shows a pronounced peak 4 with a lateral lobe 3 . For VH lobe 3 vanishes and peak 4 shifts to the right. Beam profile for $\mathrm{V} \phi$ is reconstructed by adding the VV and VH profiles (Fig. 7 (c) and 7 (d)) as occurred with $\mathrm{LiNbO}_{3}$.

Fig. 8 (a) exhibits the beam profile obtained with $\mathrm{H} \phi, \mathrm{HH}$ and $\mathrm{H}_{4} 5^{\circ}$ configurations. For $\mathrm{H} \phi$ there

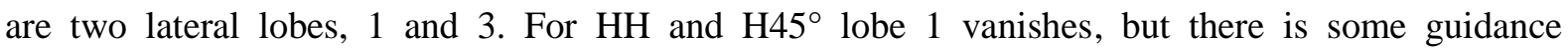
corresponding to lobe 3. Profiles at the horizontal axis, Fig. 8 (b), exhibit the intense peak 5 with two lateral lobes, 4 and 6 for all arrangements of P1 and P2. For HV in both axes, peak 2 splits in two peaks. Beam profile for $\mathrm{H} \phi$ is also reconstructed by adding the $\mathrm{HV}$ and $\mathrm{HH}$ profiles (Fig. 8 (c) and 8 (d)). 

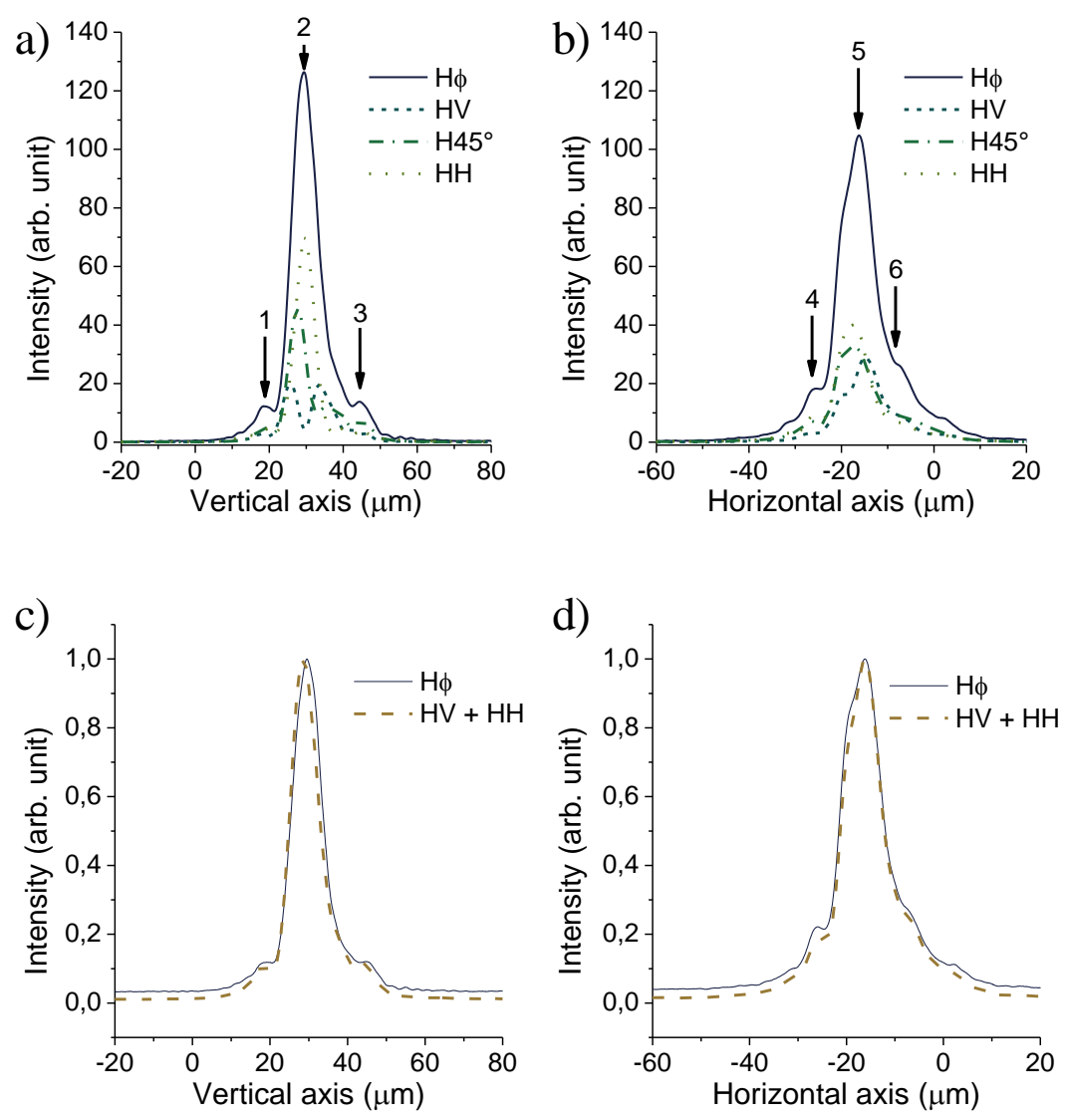

Fig. 8. (a, b) Graphical representations of the near field profile of the beam emerging from the structure inscribed in $\mathrm{LiF}$ for $\mathrm{P} 1$ at $\mathrm{H}$ direction and $\mathrm{P} 2$ absent or at $\mathrm{V}, 45^{\circ}$ and $\mathrm{H}$ directions; (c, d) Signal obtained with $\mathrm{P} 1$

$(\mathrm{H})$ and without P2 $(\Phi)$ Compared with the combination of the signals obtained in SOP in HV and HH.

\section{CONCLUSION}

The intrinsic birefringence of the bulk material influences the guidance properties of structures produced in crystals by femtosecond laser. The anisotropy caused by the highly focused writing beam superimposes an induced birefringence to the intrinsic $\mathrm{LiNbO}_{3}$ birefringence, affecting the SOP of light propagating in the structure. Even for a non-birefringent crystal, as LiF, the induced birefringence leads to changes in the SOP of the guided light.

Structures produced in $\mathrm{LiF}$ and $\mathrm{LiNbO}_{3}$ by the femtosecond laser are composed of anisotropic regions with different birefringence. Choosing adequately the writing parameters and the bulk material as well as the SOP of the coupled light, it can be constrained to propagate in a previously determined region of the crystal. Such characteristic may allow tailoring the waveguides for a specific application, e.g. an evanescent field sensor where the light propagation can be confined close to the material surface, enhancing the interaction with the surrounding medium.

\section{ACKNOWLEDGMENT}

We thank the UTFPR for support received during the work realization. This work was supported by Capes, CNPq, Fundação Araucária and FINEP/ CT-Infra. 


\section{REFERENCES}

[1] K. M. Davis, K. Miura, N. Sugimoto, K. Hirao, "Writing waveguides in glass with a femtosecond laser," Optics Letters, vol. 21, pp. 1729-1731, 1996.

[2] S. Nolte, M. Will, J. Burghoff, A. Tuennermann, "Femtosecond waveguide writing: a new avenue to three-dimensional integrated optics," Appl. Phys. A: Materials Science \& Processing, vol. 77, pp. 109-111, 2003.

[3] K. Miura, J. Qiu, H. Inouye, T. Mitsuyu and K., Hirao, "Photowritten optical waveguides in various glasses with ultrashort pulse laser," Appl. Phys. Lett., vol. 71, pp. 3329-3331, 1997.

[4] M. Ams, G.D. Marshall, P. Dekker, J.A. Piper and M.J. Withford, "Ultrafast laser written active devices," Laser Photonics Rev., vol. 3, no. 6, pp. 535-544, 2009.

[5] J. Burghoff, S. Nolte, A. Tuennermann, "Origins of waveguiding in femtosecond laser-structured $\mathrm{LiNbO}_{3}$," Appl. Phys. A: Materials Science and Processing, vol. 89, pp. 127-132, 2007.

[6] J. Burghoff, H, Hartung, S. Nolte, A. Tuennermann, "Structural properties of femtosecond laser-induced modifications in $\mathrm{LiNbO}_{3}$," Appl. Phys. A: Materials Science and Processing, vol. 86, pp. 165-170, 2006.

[7] P. Rabiei and P. Gunter, "Optical and electro-optical properties of submicrometer lithium niobate slab waveguides prepared by crystal ion slicing and wafer bonding," Appl. Phys. Lett., vol. 85, no. 20, pp. 4603-4605, 2004.

[8] R. S. Weis, T. K, Gaylord, "Lithium niobate: summary of physical properties and crystal structure," Appl. Phys. A: Materials Science \& Processing, vol. 37, pp. 191-203, 1985.

[9] I. Baumann, S. Bosso, R. Brinkmann, R. Corsini, M. Dinand, A. Greiner, K. Schäfer, J. Söchtig, W. Sohler, H. Suche, and R. Wessel, "Er-Doped integrated optical devices in $\mathrm{LiNbO}_{3}$," IEEE J. Sel. Top. Quant. Electron., vol. 2, no. 2, pp. 355-366, 1996.

[10] W. Sohler, B. K. Das, D. Dey, S. Reza, and H. Suche, "Erbium-Doped lithium niobate waveguide lasers," IEICE Trans. Electron., no. 5, pp. 990-997, 2005.

[11] R. K Choubey, P. Sen, P. K. Sen, R. Bhatt, S. Kar, V. Shukla, K. S. Bartwal, "Optical properties of MgO doped $\mathrm{LiNbO}_{3}$ single crystals," Opt. Materials, vol. 28, pp. 467-472, 2006.

[12] L. Gui, B. Xu, and T. C. Chong, "Microstructure in lithium niobate by use of focused femtosecond laser pulses," IEEE Photonics Technol. Lett., vol. 16, no. 5, pp. 1337-1339, 2004.

[13] H. Chen, T. Lv, A. Zheng, and Y. Han, "Directly writing embedded waveguides in lithium niobate by a femtosecond laser," Opt. - Int. J. Light Electron Opt., vol. 124, no. 3, pp. 195-197, 2013.

[14] R. R. Thomson, S. Campbell, I. J. Blewett, a. K. Kar, and D. T. Reid, "Optical waveguide fabrication in z-cut lithium niobate (LiNbO[sub 3]) using femtosecond pulses in the low repetition rate regime," Appl. Phys. Lett., vol. 88, pp. 111109-1 - 111109-3, 2006.

[15] S. Bhardwaj, K. Mittholiya, A. Bhatnagar, R. Bernard, J.A. Dharmadhikari, D. Mathur and A.K. Dharmadhikari, "Inscription of type I and depressed cladding waveguides in lithium niobate using a femtosecond laser," Appl. Optics, vol. 56, no. 20, pp. 5692-5697, 2017.

[16] J. Lv, X. Hao and F. Chen, "Green up-conversion and near-infrared luminescence of femtosecond-laser-written waveguides in $\mathrm{Er}^{3+}, \mathrm{MgO}$ co-doped nearly stoichiometric $\mathrm{LiNbO}_{3}$ crystal," Optics Express, vol. 24, no. 22, pp. 2548225490, 2016.

[17] J. Burghoff, C. Grebing, S. Nolte and A. Tuennermann, "Efficient frequency doubling in femtosecond laser-written waveguides in lithium niobate," Appl. Phys. A: Materials Science and Processing, vol. 89, pp. 081-108, 2006.

[18] J. Qi, P. Wang, Y. Liao, W. Chu, Z. Liu, Z. Wang, L. Qiao, and Y. Cheng, "Fabrication of polarization-independent single mode waveguides in lithium niobate crystal with femtosecond laser pulses," Optical Materials Express, vol.6, no.8, pp. 2554-2559, 2016.

[19] G. Baldacchini, "Colored LiF: an optical material for all seasons," Journal of Luminescence, vol. 100, pp. 333-343, 2002.

[20] J. Nahum and D. A. Wiegand “Optical properties of some F-aggregate centers in LiF," Phys. Rev. pp. 154 817, 1967.

[21] H. J. Kalinowski, R. M. Montereali, M. A. Vincenti and R. N. Nogueira, "Visible photoluminescence in coloured lithium fluoride under ultra-violet continuous wave excitation," J. Phys. C:Conf. Series, 249, 2010, pp. 012002.

[22] R. A. Nunes, H. J. Kalinowski, S. Paciornik, A. M. Souza, and L. C. Scavarda do Carmo, "Electron-beam production of colour centres on alkali halide crystals and films," Nucl. Instrum. Methods Phys. Res. B, vol. 32, pp. 222, 1988.

[23] R. M. Montereali, A. Mancini, G. C. Righini, and S. Pelli, "Active stripe waveguides produced by eléctron beam lithography in LiF single crystals," Opt. Commun., vol 153, pp. 223, 1998.

[24] T. Kurobori, T. Sakai, S. A. Aoshima, "A narrow band, green-red colour centre laser in LiF fabricated by femtosecond laser pulses," Phys. Stat. Sol., vol. 204a, no. 3, pp. 699-705, 2007.

[25] I. Chiamenti, F. Bonfigli, R. M. Montereali and H. J. Kalinowski, "Dimensions and refractive index estimates of deeply buried optical waveguides in lithium fluoride," J. Microw. Optoelectr. Eletromag. Appl., vol.13, no. 1, pp. 47-54, 2014.

[26] I. Chiamenti, F. Bonfigli, A. S. L. Gomes, R. M. Montereali, L. N. Costa and H.J. Kalinowski, "Broadband optical active waveguides written by femtosecond laser pulses in lithium fluoride," Chin. Phys. Lett., vol. 31, no.1, pp. 014201, 2014.

[27] ] I. Chiamenti, F. Bonfigli, A. S. L. Gomes, F. Michelotti, R. M. Montereali and H.J. Kalinowski, J. Appl. Phys vol. 115, pp. 023108, 2014.

[28] T. Kurobori, K. Inabe, and N. Takeuchi, "Room temperature visible distributed-feedback colour centre laser," J. Phys. D: Appl. Phys., vol. 16, no. L121, 1983.

[29] K. Kawamura, M. Horano, T. Kurobori, D. Takamizu, T. Kamiya, and H. Hosono, "Femtosecond-laser-encoded distributed-feedback color center laser in lithium fluoride single crystals," Appl. Phys. Lett., vol. 84, no. 3, pp. 311, 2004. 
[30] M. A. Vincenti, S. Almaviva, R. M. Montereali, H. J. Kalinowski, and R. N. Nogueira, "Permanent luminescent micropatterns photoinduced by low-power ultraviolet irradiation in lithium fluoride," Appl. Phys. Lett., Vol. 89, pp. $241125,2006$.

[31] R.M. Montereali, F. Bonfigli, E. Nichelatti, M.A. Vincenti, "Versatile lithium fluorde thin-film solid-state detectors for nanoscale radiation imaging," Nuovo Cimento C, vol 36, no. 2, pp. 35, 2013.

[32] V. V. Ter-mikirtychev, "Stable room-temperature LiF:F2+ tunable color-center laser for the 830-1060-nm spectral range pumped by second-harmonic radiation from a neodymium laser," Applied Optics, vol. 34, no. 27, pp. 6114-6117, 1995.

[33] Y. Obayashi, et al. "Red and green distributed-feedback LiF color center lasers fabricated by interference of femtosecond laser pulses," The Review of Laser Engineering, vol. 36, pp. 1226- 1229, 2008.

[34] M. Sakakura, Y. Shimotsuma, N. Fukuda, K. Miura, "Transient strain distributions during femtosecond laser-induced deformation inside LiF and MgO single crystals,” Journal of Applied Physics, vol. 118, 023106, 2015. 